\title{
Relación entre la ocupación y la automedicación en adultos mayores de 65 años en adelante, en la parroquia el Sagrario, Cuenca-Ecuador, 2017.
}

\section{Relationship between occupation and self-medication in adults over 65 years of age, El Sagrario parish, Cuenca-Ecuador, 2017.}

\author{
Bravo - Angamarca Lourdes ${ }^{1 *}$, Idrovo - Iñiguez Eugenio ${ }^{2}$ Pesantes Eduardo $^{3}$ \\ ${ }^{1}$ Universidad Católica de Cuenca-Ecuador \\ *alexbravo_1995@hotmail.com
}

\begin{abstract}
Resumen
Relacionar la ocupación con la automedicación en adultos mayores de 65 años de edad en la parroquia el Sagrario, CuencaEcuador, 2017. La metodología aplicada fue de tipo caso-control porque se buscó asociar la relación entre 2 variables, la ocupación y automedicación. Utilizando la encuesta dirigida como instrumento se realiza una investigación de campo. La población estuvo conformada por 801 personas mayores de 65 años de edad que residen en la parroquia El Sagrario, se obtuvo una muestra de 417 personas que representan la totalidad de la población. La misma que fue calculado considerando la diferencia entre los dos grupos de estudio concluyendo un tamaño muestral de 116 personas, constituida a su vez por 58 personas en casos y 58 personas en los controles. Para determinar la exposición al factor de riesgo se obtuvo 47 casos y 42 controles expuestos, y 11 casos y 16 controles no expuestos. Se reporta que el Odds Ratio es de 1.628 con IC de $(0 ; 4)$ por lo tanto se interpreta que la ocupación no tiene asociación con la automedicación y es un factor de protección.
\end{abstract}

Palabras clave: Automedicación, ocupación, adulto mayor.

\begin{abstract}
Relate occupation with self-medication in adults over 65 years of age in El Sagrario parish, Cuenca-Ecuador, 2017. The applied methodology was the case control type because it looked for the relationship between 2 variables, occupation and self-medication. A field investigation was carried out, using the survey as an instrument. The population consisted of 801 people over 65 years of age residing in the El Sagrario parish, resulting in a total representative sample of the population of 417 people. The size of the sample has been calculated based on the difference between the two groups and is composed of 116 people, consisting of 58 people in cases and 58 people in the controls. To determine the risk factor exposure, 47 cases and 42 exposed controls were obtained, and 11 cases and 16 controls not exposed. It is reported that the Odds Ratio is 1,628 with CI of $(0 ; 4)$ therefore it is interpreted that the occupation has no association with self-medication and is a protective factor.
\end{abstract}

Key words: Self-medication, occupation, elderly.

\section{INTRODUCCIÓN}

El propósito del Plan Nacional de Salud es garantizar a la población servicios que ofrezcan atención de calidad, con el desarrollo de actividades de promoción, educación, prevención, diagnóstico y tratamiento de diferentes patologías. Sin embargo, no se está cumpliendo íntegramente ya que ha entrado en controversia específicamente al referirse al tratamiento y diagnóstico de las enfermedades por la autonomía que han adoptado las personas sobre su propia salud1. La selección y uso de medicamentos que frecuentemente utilizan las personas con el propósito de tratar enfermedades leves o síntomas que ellos mismos creen identificar es definido por la OMS como la Automedicación. Este fenómeno ha llegado a ser promovido como una forma de autocuidado, el cual ha recibido en cierto grado un impacto positivo, logrando establecer claramente sus ventajas en la disminución de gastos de los diversos sistemas de salud, pero también cabe señalar la preocupación que esta acción provoca como la potencialidad a la aparición de efectos no deseados vinculados con diagnósticos terapéuticos inadecuados. ${ }^{1}$

La OMS determino que más del $50 \%$ de la prescripción de los medicamentos se prescriben inapropiadamente en Latinoamérica; el autoconsumo de fármacos en la actualidad se ha convertido en un problema grave de salud pública, las causas más frecuentes nacen de la desconfianza que existe hacia los médicos y odontólogos y de las deficiencias que existe 
en los sistemas de salud como la poca cobertura y alto costo de los fármacos, de hecho, una particularidad que existe en nuestro país y que día a día cobra fuerza es la crisis económica que enfrentan las personas adultas mayores generado por el desempleo o por la incapacidad de ejercer un trabajo dando lugar a una inestabilidad económica. ${ }^{2}$ Actualmente no existe suficiente información que nos permitan determinar que la actividad laboral es un factor de riesgo que desencadene automedicación, por lo que no existe elementos de juicio que nos permitan establecer estrategias para minimizar este problema, esta es la razón por la que mediante un estudio caso-control, utilizando como instrumento la encuesta de Andrea Conhi, se pretende relacionar la ocupación con la automedicación en adultos mayores de 65 años de edad para clarificar la doble percepción con que se relaciona este fenómeno y a su vez determinar cuál de ellos tiene mayor relevancia social.

\section{MARCO TEÓRICO}

\subsection{El adulto mayor en la sociedad}

Actualmente existe un gran incremento del número de ciudadanos que tienen más de 60 años, en América Latina se ha demostrado que existe aproximadamente 32 millones de personas que tienen 60 años o más, de este porcentaje el $53.4 \%$ son mujeres, pero esta cantidad no es estable ya que va incrementándose anualmente en un $3 \%$. En el Ecuador según datos del INEC hay 14.483.499 habitantes, de ellos 940.905 son mayores de 65 años de edad, es decir el $6.5 \%$ de la población total nacional son personas adultas mayores, en cuanto a la distribución por sexo obtenemos un $53 \%$ para las mujeres y un $47 \%$ para los hombres. ${ }^{4}$ En la provincia del Azuay de acuerdo a los datos extraídos del último censo realizado en el 2010 existe 55.835 adultos mayores representando el $8 \%$ del total de la población y en la ciudad de Cuenca aproximadamente 35.819 adultos mayores a 65 años de edad, porcentualmente equivale al $10.79 \%$ de la población total de la ciudad. Y es precisamente este incremento lo que ha ocasionado la aparición de debates públicos sobre temas como mejoramiento de la calidad, nivel de seguridad y supervivencia en relación con las acciones y reacciones del entorno, interacción con la sociedad, atención de la salud y otros. 4

\subsection{Automedicación en el adulto mayor}

El término automedicación está incluido en el tesauro de la Nacional Library of Medicine de Estados Unidos y es definido como la utilización de medicamentos por iniciativa propia para tratar sintomatología o enfermedades sin ninguna intervención por parte del médico ni en el diagnóstico de la enfermedad, ni en la prescripción o supervisión del tratamiento. Un aspecto interesante del término automedicación es apreciar su doble origen, por un lado, se deriva de "drug therapy" que significa terapia farmacología y por el otro de "self care" que se refiere a autocuidado. ${ }^{3}$ Esta doble derivación que se le atribuye tiene una apreciación desde el punto de vista de sus implicaciones prácticas y éticas, es decir, si nos referimos a automedicación responsable, es decir, bajo criterio formado en educación en Salud, se lo considera como una práctica responsable, sin embargo, si se relaciona con el consumo de medicamentos por iniciativa propia, podría considerarse como una desviación de la terapia farmacológica. ${ }^{5}$

Uno de los claros e inevitables acontecimientos por los que atraviesan las personas de la tercera edad es el deterioro progresivo de su organismo, lo que origina la aparición de diversas enfermedades. Estas inestables condiciones son la causa principal que genera el consumo de medicamentos, cabe recalcar que estos a veces no son prescritos por el profesional de la Salud, provocando en algunos casos que el problema se solucione y la mayoría de las ocasiones provoque reacciones adversas5. Las personas adultas mayores necesitan los servicios de salud con prioridad, sin embargo, la accesibilidad a estos servicios es limitada, esto puede deberse a la presencia de algún tipo de discapacidad o por las limitaciones de la edad y la tendencia de consumir fármacos por iniciativa propia. Al contrario de los establecimientos farmacéuticos, que, por la facilidad a su acceso, bajo costo y el conocimiento que se pretende tener sobre los fármacos más utilizados, conocimiento que se limita solamente a la acción que ejercen sobre el organismo, ya que se omite la importancia de conocer los efectos adversos que ocasionan, las dosis eficaces para causar el efecto deseado5. La existencia de errores en el consumo de medicamentos como tomar la dosis incorrecta, abandonar el tratamiento farmacológico, reutilizar y no verificar etiquetas; combinar fármacos con alcohol, entre otros errores frecuentes, se debe a una falta en la comprensión de las normativas en la administración de medicamentos. 6

\subsection{Relación de la ocupación con la automedicación en} adultos mayores.

En américa Latina el envejecimiento es la etapa más proclibe a situaciones de pobreza, la persistente inequidad social, creciente participación laboral en el mercado informal, a más de estos se suma las dificultades socio-económicas, desigualdad de género y discriminación étnica. Las malas condiciones de trabajo o el desempleo, dificulta y a la vez disminuye la accesibilidad a los centros de salud, provocando que las personas busquen otro tipo de alternativas, recurriendo a la automedicación. ${ }^{6}$ En el 2007 el Ministerio de Salud Pública (MSP) promulgó Políticas de Salud, enfocados en promover, conservar y recuperar la salud de los adultos mayores, además actualmente establece unidades operativas de los tres niveles de atención que permiten el acceso a la asistencia continua y gratuita. Con el propósito de fortalecer la calidad de vida de las personas de la tercera edad, el Instituto Ecuatoriano de Seguridad Social (IESS) también brinda atención a través de programas de salud integral a los afiliados, jubilados y beneficiarios inscritos, y es el Seguro Social Campesino (SSC) que protege aproximadamente a un $23 \%$ de la población adulta mayor del sector rural. ${ }^{6}$ 
Actualmente el MSP y el IESS brindan servicios de salud a 625.001 adultos mayores, y desde el 2007 hasta la actualidad se han diseñado y puesto en marcha programas que acogen a la población adulta mayor como el Programa de Atención Integral Gerontológica (2011), La Red de Protección Solidaria (2008), El Proyecto Adulto Mayor (2009), La Propuesta de Estándares de Calidad de Atención al Adulto Mayor (2011) y El Programa de Dirección Provincial de Gerontología (2011) Se ha priorizado atención y se ha incorporado la participación activa en la sociedad, considerándolos como grupo vulnerable. El establecer la relación de la automedicación con la ocupación que desempeñan los adultos mayores, puede ser apreciada desde diversos puntos de vista.

1. Toda persona independientemente de que ejerza un trabajo o no, al sentir dolor, malestar, o presentar algún cuadro patológico que genere enfermedad consume medicamentos por iniciativa propia por la facilidad de su acceso. ${ }^{7}$

2. Los adultos mayores tienden a practicar automedicación con frecuencia, en algunos casos para evitar caer enfermos y poder desempeñar adecuadamente sus actividades diarias. $^{2}$

3. En ocasiones las malas condiciones de trabajo y el bajo ingreso económico impiden al adulto mayor acceder a los diferentes servicios de salud y se ven en la necesidad de autoprescribirse fármacos para aliviar el dolor o malestar que padecen. $^{4}$

4. Actualmente existe una cobertura a los servicios de salud para las personas mayores que trabajan, respondiendo a sus necesidades, promoviendo conductas de autocuidado a través de leyes, políticas, programas y acciones nacionales y comunitarias. $^{7}$

5. Cada vez existen más recursos humanos que facilitan la implementación de diversos programas que velan por el bienestar de los adultos mayores con énfasis en el nivel de atención primaria por lo que el índice de automedicación disminuye. $^{4}$

6. La existencia de instituciones y/o personas que trabajan con objetivos comunes y en estrecha coordinación ha hecho posible que las personas adultas mayores reciban atención prioritaria y especializada en ámbitos públicos y también privados sobre todo en el campo de la salud por lo que la frecuencia de consumo de fármacos por iniciativa propia disminuye. $^{7}$

\section{MATERIALES Y MÉTODOS}

Para alcanzar el objetivo propuesto se ha llevado a cabo un estudio caso-control, retrospectivo para identificar el papel del posible factor de riesgo atribuible a la automedicación en adultos mayores de 65 años en el Sagrario de la ciudad de Cuenca, efectuando un diseño pareado uno a uno, por edad y sexo. 8 El universo se conformó, definiendo como criterios de inclusión a toda persona residente de la parroquia el Sagrario del cantón Cuenca, que cumplía con el rango de edad de 65 años en adelante, se excluyeron a aquellas personas cuya profesión era de médico, odontólogo o enfermera, personas que no firmaron el consentimiento y personas que presentaron alguna condición psíquica o mental perceptible que les imposibilito a comunicarse y contestar con veracidad.

Para saber cuál es el tamaño muestral y a cuantas personas incluir en el estudio se aplicó formula, obteniendo como resultado una muestra de 417 personas. El método de muestreo de estuduo es de caso-control y la muestra se calculó en base a la diferencia de cada grupo. Posterior a la aplicación de la fórmula en el programa de libre acceso Open-Epi, se concluye un tamaño muestral de 116 personas, constituida a su vez por 58 personas en casos y 58 personas en controles. ${ }^{9,10}$

Se utilizó la técnica de la encuesta dirigida a los participantes en la investigación para la recolección de información con previo consentimiento informado. Para ello se utilizó el cuestionario de encuesta de Andrea Conhi, la cual incluye dos secciones: ${ }^{11}$ La primera parte compuesta por los datos generales del encuestado (nombre, edad, sexo, ocupación, nivel de instrucción, ingreso económico) y la segunda parte constituida por Ítems de respuesta múltiple, para evaluar la frecuencia de automedicación.

Una vez verificado que el encuestado firmó el consentimiento informado, se procedió a realizar el cuestionario, solicitando la contestación de los ítems planteados, y finalizada su aplicación, se registraron en una base de datos en el programa Epi-Info, consignando ordenadamente los datos. Para el tratamiento y análisis de datos se utilizó el programa estadístico de acceso libre Open-Epi y Microsoft office Excel y para la descripción de los datos cuantitativos, se utilizó tablas que permitieron sintetizar su distribución. Finalmente, para determinar las relaciones entre las variables del estudio se realizó el cálculo basado en Odds Rattio, obteniendo un valor de 1.621, un nivel de confianza de dos lados del $95 \%$, una potencia del $80 \%$ y un nivel de significancia estadística del $5 \%(\mathrm{p}<0.05)$

\section{RESULTADOS}

El estudio se realizó con los datos obtenidos de la parroquia el Sagrario, mostrando los siguientes resultados:

\begin{tabular}{ccccccc}
\hline & \multicolumn{2}{c}{ HOMBRES } & \multicolumn{2}{c}{ MUJERES } & \multicolumn{2}{c}{ TOTAL GENERAL } \\
& $\mathrm{n}$ & $\%$ & $\mathrm{n}$ & $\%$ & $\mathrm{n}$ & $\%$ \\
\hline CASOS & 32 & $50 \%$ & 26 & $50 \%$ & 58 & $50 \%$ \\
CONTROLES & 32 & $50 \%$ & 26 & $50 \%$ & 58 & $50 \%$ \\
Total & 64 & $55 \%$ & 52 & $45 \%$ & 116 & $100 \%$ \\
\hline
\end{tabular}

Fig. 1. Tabla 1. Distribución de la muestra de acuerdo al sexo

Tabla 1. En esta tabla se puede evidenciar que la distribución de acuerdo al sexo coincide tanto en casos como en controles, realizado mediante la estrategia de emparejamiento. Así tenemos que de un hombre (caso) con un hombre (control) se obtuvo un total de 32 casos y 32 controles, y el 
emparejamiento de una mujer (caso) con una mujer (control) se obtuvo 26 casos y 26 controles.

\begin{tabular}{ccccccc}
\hline & \multicolumn{2}{c}{ CASOS } & \multicolumn{2}{c}{ CONTROLES } & \multicolumn{2}{c}{ TOTAL } \\
& $\mathrm{N}$ & $\%$ & $\mathrm{~N}$ & $\%$ & $\mathrm{n}$ & $\%$ \\
\hline Expuestos & 47 & $53 \%$ & 42 & $47 \%$ & 89 & $77 \%$ \\
No expuestos & 11 & $41 \%$ & 16 & $59 \%$ & 27 & $23 \%$ \\
Total & 58 & $50 \%$ & 58 & $50 \%$ & 116 & $100 \%$ \\
\hline
\end{tabular}

Fig. 2. Tabla 2. Distribución de la muestra de acuerdo a la edad

Tabla 2. En esta tabla se puede evidenciar que la distribución de acuerdo a la edad coincide tanto en casos como en controles, realizado mediante la estrategia de emparejamiento. Así tenemos que la edad más sobresaliente fue de 65 años, registrándose 12 casos y 12 controles.

\begin{tabular}{lcccccc}
\hline & \multicolumn{2}{c}{ CASOS } & \multicolumn{2}{c}{ CONTROLES } & \multicolumn{2}{c}{ TOTAL } \\
& $\mathrm{N}$ & $\%$ & $\mathrm{~N}$ & $\%$ & $\mathrm{n}$ & $\%$ \\
\hline Expuestos & 47 & $53 \%$ & 42 & $47 \%$ & 89 & $77 \%$ \\
No expuestos & 11 & $41 \%$ & 16 & $59 \%$ & 27 & $23 \%$ \\
Total & 58 & $50 \%$ & 58 & $50 \%$ & 116 & $100 \%$ \\
\hline
\end{tabular}

Fig. 3. Tabla 3. Relación entre la automedicación y la ocupación

Tabla 3. En esta tabla se puede observar que para determinar la exposición al factor de riesgo se obtuvo 47 casos y 42 controles expuestos, y 11 casos y 16 controles no expuestos. Se reporta que el Odds Ratio es de 1.628 con IC de $(0 ; 4)$ por lo tanto se interpreta que la ocupación no tiene asociación con la automedicación y es un factor de protección.

\section{DISCUSIÓN}

La presente investigación determinó que la exposición no tuvo asociación con la enfermedad, es decir, la ocupación de los adultos mayores de 65 años de edad de la parroquia el Sagrario de la ciudad de Cuenca, no es factor de riesgo de la automedicación. El estudio realizado en esta zona urbana del cantón Cuenca, constó de una muestra de 116 personas, se incluyeron en el estudio 58 personas como casos y 58 personas como controles. Y en el análisis de la distribución de la muestra obtenida según la edad y el sexo se realizado mediante la estrategia de emparejamiento, coincidiendo tanto en casos como en controles.

Evidentemente existen muchas investigaciones realizadas que determinan la prevalencia de automedicación en el Ecuador, sin embargo, estudios caso-control que relacionen la actividad laboral de las personas con la automedicación son pocas, por lo que el presente estudio ejecutado en la ciudad de Cuenca incentiva la realización de nuevas investigaciones que abarquen otros grupos poblacionales, y de esta manera poder establecer una discusión en base a los resultados.

Se realizó comparaciones con otros autores, a pesar de que estamos conscientes de las diferencias que tiene nuestro estudio con otros, como, por ejemplo, el tamaño muestral, la ubicación espacial, nivel sociocultural, criterios de selección de los participantes, diseño de la investigación, enfoque y temporalidad del estudio, análisis multivariado de correlación de variables, técnicas de recolección de información, codificación, tabulación y análisis estadísticos, entre otros.

López y Bermúdez (Colombia 2015) afirman que personas con o sin ocupación ante el dolor recurren al consumo de fármacos por iniciativa propia12, Willis Slattery (Italia 2016) establecen que la edad y el sexo no son razones para automedicarse13 y Escobar (México 2011) determino que la edad, sexo, nivel sociocultural y situación laboral no son razones para automedicarse. ${ }^{14}$ Otros autores sostienen criterios diferentes al del este estudio como Mestanza y Pamo (Brazil 2015) quienes afirman que la ocupación si influye en la automedicación, ${ }^{15}$ además Rivera Espino (Perú 2012) afirma que el desempleo es una razón para automedicarse16 y finalmente Muñoz (Ecuador 2012) quien reconoce que el desempleo no permite un adecuado acceso a la salud. ${ }^{17}$

\section{CONCLUSIONES}

El análisis de la distribución de la muestra obtenida según la edad y el sexo de los adultos mayores de 65 años de edad de la parroquia El Sagrario, realizado mediante la estrategia de emparejamiento, coincide tanto en casos como en controles. El estudio reporto un Odds Rattio de 1.628, lo que se interpreta que la exposición, en este caso la ocupación no tuvo asociación con la automedicación, dado que el intervalo de confianza es $0 ; 4$ se considera que no existe evidencia suficiente a favor de la asociación de las variables. Resultado que concuerda con la hipótesis establecida ya que la ocupación no es factor de riesgo, sino factor de protección de la automedicación. Las razones por la que la ocupación no es factor de riesgo de la automedicación, probablemente sean las siguientes:

1. Porque la obtención de los datos para el estudio fue realizada en una sola parroquia de la ciudad de Cuenca, y el tamaño muestral obtenido era pequeño. 2. Porque gracias a las actuales políticas de salud integral gerontológica, disminuye la necesidad de los adultos mayores de tratar por iniciativa propia sus propias dolencias, por lo tanto, disminuye la automedicación. 3. Porque actualmente el MSP y las empresas farmacéuticas restringen la venta de medicamentos que podrían ocasionar problemas graves. 4 . Porque en este grupo etario la ocupación es relativa en cuanto a la remuneración.

\section{Referencias Bibliográficas}

1 Proceso de Normalización del SNS. In Federación Odontológica Ecuatoriana; 2009 jun.

2 Molina-Proaño G, Tello-Echanique G. Factores asociados a la automedicación en profesionales de salud. Quito: Pontificia Universidad Católica del Ecuador, Facultad de Medicina; 2013 Sep. 
3 Domínguez-Torres R, Hechavarria-Espinosa A. Polifarmacia en la tercera edad. Algunas consideraciones. 2010 Octubre; 3(2).

4 Chacha-Gualli P. Promoción de estilos de vida saludables en el club de adultos mayores de la providencia. Cantón Guano. Tesis de Grado. Riobamba: Escuela Superior Politécnica de 20 Chimborazo, Facultad de Salud Pública; 2015.

5 Chávez-Alayo F. Nivel de conocimientos básicos sobre medicamentos, nivel educativo y automedicación en pacientes 2 del hospital La Caleta de Chimbote. Disponible en: Maestría de investigación. Universidad Inca Garcilaso de la Vega; 2 2016.

6 Agenda de Igualdad para Adultos mayores. Ministerio de Inclusión Económica y Social, Constitución de la República del Ecuador; 2012.

7 Aguedo-Castillo A, Chein-Villacampa S. Relación entre la automedicación para el manejo del dolor dentario y el 24 nivel socioeconómico-cultural de los pobladores del distrito de Independencia Huaraz.Rev. Investig. UNMSM. 2012; 11(2).

8 Villavicencio Caparó E, Alvear Córdova. M, Cuenca León 25 K, Calderón Curipoma M, Palacios Vivar D. Diseños de estudios clínicos en odontología. Revista OACTIVA UC Cuenca.2016; Vol. 1, No. 2, pp. 83-86.

9 Villavicencio-Caparó E. El tamaño muestral en tesis de post grado. ¿Cuantas personas debo encuestar? Conference 27 Aniversario de la Escuela de Postgrado, At Universidad Católica de Santa María Arequipa. Enero 2011.

10 Villavicencio-Caparó E. El diseño de Casos y Controles. 28 Universidad Peruana Cayetano Heredia. Postgrado en Estomatologia. 2000

11 Conhi A, Castillo D, López Cesar. Automedicación odontológica de pacientes que acuden a una institución pública y a 29 una privada, Lima-Perú. Rev.Estomat. Heredi.2015; 25(3). .

12 Lopez-Cabra C. Automedicación en estudiantes de medicina de la Universidad del Rosario en Bogotá D. C., Colom- 3 bia. Rev Colomb. Cienc Quim. Farm. 2015; 45 (3).

13 Willis S, Slattery A. King W. Llamadas de error terapéutico entre adultos mayoresreportados a un Centro Regional. South Med J. 2016 ; 109 (6)

14 Escobar-Toledo A. Determinacion de las razones y diferen- 3 cias en automedicación entre una parroquia urbana u una parroquia rural del Distrito de Quito. May. 2011.

15 Mestanza S. Extensión universitaria: un enfoque desde el 3 campo de la salud. En Revista Masquedós. 2015; 2 (2).

16 Rivera-Espino O, Urcia-Pelaez J. Nivel de Conocimiento, Actitudes y ResultadosNegativos de la automedicación en 33 estudiantes de la universidad Pública.Lambayeque 20112012. Rev. Cuerpo Médico. HNAAA.2012; 10 (1).

17 Muños J, Oscanoa T, Fernández M. Utilización y accesibi- 34 lidad a medicamentos en el Sistema Nacional del Seguro Social. 2012.

18 Regueira-Betancourt S, Rodríguez-Fernández A. Interven- 35 ción sobre la automedicación en el Consultorio Medico de la
Familia. Revista Electrónica Zoilo E. Marinello Vidairreta. 2012; 38(4).

19 Junta de la Castilla y León. Medicamentos sin receta y automedicación responsable. In Sacylipe; 2016; EEUU. p. $1-2$.

20 Taboada-Aranza O. Perfil de salud bucodental en un grupo de adultos mayores del estado de Hidalgo. ADM. 2014; 71(2).

21 Hernández-Juyol M, Boj-Quesada J. Odontología y automedicación: un reto actual. Medicina Oral. 2012; 7:334-7.

22 Tobón-Marulanda F, Montoya-Pavas S. Automedicación Familiar, un problema de salud pública. Elsevier-España. EDUMED.158; 2017.

23 Waldo-Vergara J, Solís G. Automedicación en clubes de adulto mayor de la ciudad de Valpara. Rev.Salud Publica. 2014; 18 (3).

24 Casas-Vásquez P, Ortiz-Saavedra P. Estrategias para optimizar el manejo farmacológico en el adulto mayor. Rev. Peruana de Medicina Experimental y Salud Publica. 2016; 33 (2).

5 Quispe-Pariona J. Características de la automedicación en el adulto mayor. Centro de Salud Carmen Alto - Ayacucho. Repositorio Institucional UAP. 2016.

26 Serra-Urra M, German-Meliz J. Polifarmacia en el adulto mayor. Rev Haban Cienc Med. 2013; 12(1).

27 Culquitante-Calviño L, Casas-Gross S. Efecto cascada en el anciano como consecuencia de la polifarmacia. Medisan. 2011; 21 (3).

8 Cervantes-Liñán L. Nivel de conocimientos básicos sobre medicamentos, nivel educativo y automedicación en pacientes del hospital la caleta de Chimbote. Universidad Inca Garcilaso de la Vega. 2016.

9 Ileana C, Castañeda A. Diferencias relacionadas con la salud de mujeres y hombres adultos mayores. Revista Cubana de Medicina General Integral 2013.

30 Salazar J, Bello L, Toledo A. Comportamiento epidemiológico de la automedicación y polifarmacia en individuos adultos del sector Dalia de Fernández, Municipio San Francisco. Archivos Venezolanos de Farmacología y Terapéutica. 2013; 1 (15).

1 Aponte-Daza V. Calidad de vida en la tercera edad. La PazBolivia. Red de Revistas Científicas de América Latina, el Caribe, España y Portugal. 2015; 13

2 Ureña-Guachizaca P. Conductas de consumo de medicamentos y factores familiares en el adulto mayor. Zona 7. 2016.

3 Castro-Posligua A. Medicamentos potencialmente inadecuados y su manifestación en adultos. Mayores atendidos en el hospital IESS de Babahoyo, Ecuador. 2017; 14 (44).

4 Olmo-Torres M. Automedicación y Abuso de Fármacos en la Tercera edad a propósito de un Caso Clínico. Universidad de Jaen. 2014.

5 Morillo-Narváez A. Estrategias para disminuir la automedicación en adultos mayores del centro de salud $n^{\circ} 1$, Tulcán. 
Universidad Regional Autónoma de los Andes. 2016.

36 Encalada-Ortiz C, Ortega-Tuapante J. Prevalencia y factores asociados a la automedicación con aines en adultos mayores en las parroquias urbanas de Cuenca, 2014. Universidad de Cuenca. 2015.

37 Velez-Paccha Y. Factores determinantes en la automedicación de los habitantes del barrio los almendros de la parroquia Timbara cantón Zamora. Universidad Nacional de Loja área de la salud humana. 2015.

38 Campo-Chávez N. Factores demográficos y socioeconómicos que se relacionan con la automedicación en la población rural del distrito de Chota. Universidad nacional de Cajamarca. 2014

39 Álvarez-Acosta J. Estudio sobre los factores que inciden en el hábito de la automedicación en los habitantes del barrio Policarpa Salavarrieta - etapa 3 - municipio de Apartadó Antioquia. 2013

40 Moran- Sánchez F. Estrategias efectivas a familiares o cuidadores acerca de cuidados personales, psicológicos, y espirituales que influye en el adulto mayor centro de atención integral "Santa Elena" 2012-2013.

41 Galazzi A, Lusignani M. Galazzi A, Lusignani M. Actitudes hacia la polifarmacia yla abstinencia de medicamentos entre pacientes mayores en Italia. Epub. 2016Mar Epub. 2016 Mar 7.

Recibido: 27 de junio del 2020

Aceptado: 02 de agosto del 2020 